\title{
Investigating the Binarity of S0-2: Implications for Its Origins and Robustness as a Probe of the Laws of Gravity around a Supermassive Black Hole
}

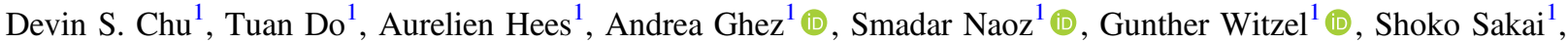 \\ Samantha Chappell ${ }^{1}$, Abhimat K. Gautam ${ }^{1}$, Jessica R. Lu ${ }^{2}$ (1) and Keith Matthews ${ }^{3}$ \\ ${ }^{1}$ Department of Physics and Astronomy, University of California, Los Angeles, CA 90095, USA \\ ${ }^{2}$ Astronomy Department, University of California, Berkeley, CA 94720, USA \\ ${ }^{3}$ Division of Physics, Mathematics, and Astronomy, California Institute of Technology, Pasadena, CA 91125, USA \\ Received 2017 September 14; revised 2017 December 18; accepted 2017 December 18; published 2018 February 6
}

\begin{abstract}
The star S0-2, which orbits the supermassive black hole (SMBH) in our Galaxy with a period of 16 years, provides the strongest constraint on both the mass of the SMBH and the distance to the Galactic center. S0-2 will soon provide the first measurement of relativistic effects near a SMBH. We report the first limits on the binarity of S0-2 from radial velocity $(\mathrm{RV})$ monitoring, which has implications for both understanding its origin and robustness as a probe of the central gravitational field. With $87 \mathrm{RV}$ measurements, which include 12 new observations that we present, we have the requisite data set to look for RV variations from S0-2's orbital model. Using a Lomb-Scargle analysis and orbit-fitting for potential binaries, we detect no RV variation beyond S0-2's orbital motion and do not find any significant periodic signal. The lack of a binary companion does not currently distinguish different formation scenarios for S0-2. The upper limit on the mass of a companion star $\left(M_{\text {comp }}\right)$ still allowed by our results has a median upper limit of $M_{\text {comp }} \sin i \leqslant 1.6 M_{\odot}$ for periods between 1 and 150 days, the longest period to avoid tidal break-up of the binary. We also investigate the impact of the remaining allowed binary system on the measurement of the relativistic redshift at S0-2's closest approach in 2018 . While binary star systems are important to consider for this experiment, we find that plausible binaries for S0-2 will not alter a $5 \sigma$ detection of the relativistic redshift.
\end{abstract}

Key words: Galaxy: center - gravitation - stars: early-type - techniques: high angular resolution

Supporting material: machine-readable table

\section{Introduction}

The source S0-2 is one of the most well-studied stars at the Galactic center. It is important for our understanding of the properties of the Galaxy's central potential. In particular, it has provided proof of the existence of a supermassive black hole (SMBH), the characterization of the SMBH properties (mass and distance), and the laws of gravitation (Ghez et al. 2005, 2008; Gillessen et al. 2009b, 2017; Boehle et al. 2016; Hees et al. 2017). S0-2 is also notable because spectroscopic studies have revealed that it, along with most of the "S-stars" located within 1 " of the black hole, is a young main-sequence B star (Ghez et al. 2003; Eisenhauer et al. 2005; Martins et al. 2008; Habibi et al. 2017). This discovery raised questions about their formation mechanism, since traditional star formation would be disrupted by the tidal forces of the black hole (Morris 1993).

Works have investigated the ways through which the S-stars may have formed and how these stars relate to the rest of the nuclear star cluster. Many theories for the S-stars' formation have been proposed (see Alexander 2005, for a review). Two general classifications of mechanisms are considered for the S-stars: (1) binary star systems scattered from outside the region and then tidally disrupted, leaving behind one component of the original binary while the other was ejected as a hypervelocity star (Hills 1988; Perets et al. 2007); and (2) S-stars formed in the clockwise disk and then migrated to the central arcsecond around the SMBH (Levin 2007; Löckmann et al. 2008; Merritt et al. 2009). Previous works have also investigated how these S-stars relate to the clockwise disk, Wolf-Rayet stars, G2-like sources, and evolved giants in the region (Paumard et al. 2006;
Bartko et al. 2009; Do et al. 2009, 2013; Lu et al. 2009; Phifer et al. 2013; Chen \& Amaro-Seoane 2014; Madigan et al. 2014; Witzel et al. 2014, 2017).

With binary stars playing a leading role in many of the S-star formation and evolution scenarios, as well as in scenarios of other Galactic center stars, observational searches for binaries are important. Thus far, photometric variations have been the primary search method. Several binaries have been revealed (Ott et al. 1999; Martins et al. 2006; Rafelski et al. 2007; Pfuhl et al. 2014), although none among the S-stars. However, eclipsing binaries are expected to be only a small fraction of the true binary population. With radial velocity (RV) measurements that now span more than a decade, there is an opportunity to search for RV variations in the brightest S-star cluster members.

Furthermore, S0-2 will be at its closest approach to the SMBH in 2018, which provides the opportunity to measure the relativistic redshift in S0-2's RV (Zucker et al. 2006; Angélil \& Saha 2010, 2011; Hees et al. 2017). This first direct observation of a relativistic effect on S-stars orbit will improve with time after 2018 and be followed by other relativistic measurement such as the advance of the periastron. If S0-2 is actually a spectroscopic binary, it will bias the relativistic redshift measurement if binarity is not considered.

In this work, we explore the possibility that $\mathrm{S} 0-2$ is a spectroscopic binary. This paper is organized as follows. In Section 2 we describe the observations and data used in this work, including new RV measurements. Section 3 describes the search for a companion star and the characterization of allowed hidden companions. Section 4 describes the impact that allowed spectroscopic binaries would have on the relativistic redshift 
Table 1

Summary of Keck Spectroscopic Observations

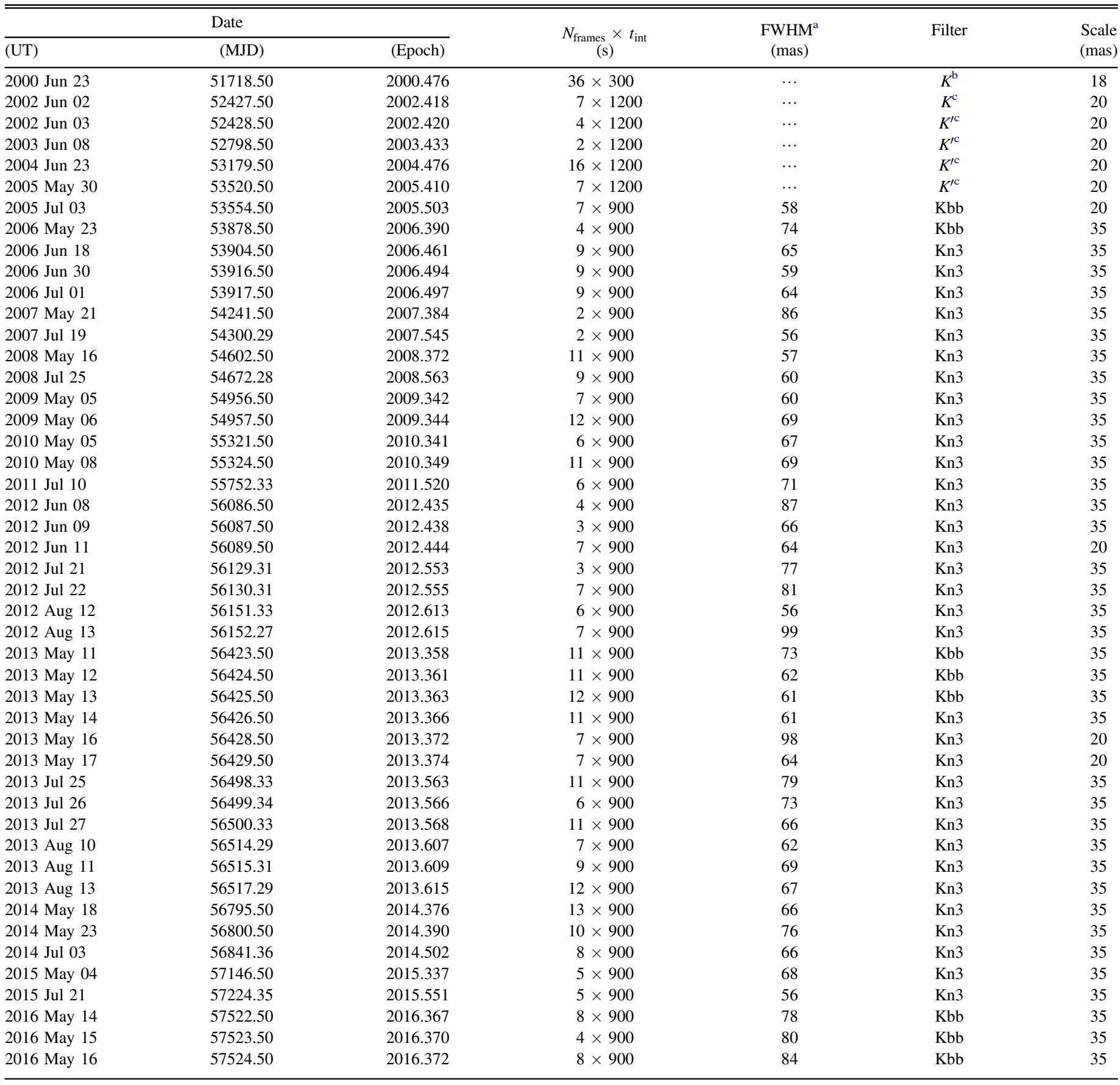

Notes.

${ }^{\text {a }}$ Average FWHM of S0-2 in the mosaic mode of all frames, measured by fitting a two-dimensional Gaussian to the source.

$\mathrm{b}$ Taken with the NIRSPEC slit spectrograph.

${ }^{\mathrm{c}}$ Taken with the NIRC2 slit spectrograph.

measurement. Section 5 interprets the results of the analysis and implications for S0-2 being a single star and the robustness of gravitational redshift measurements and future relativity studies based on S0-2's orbital motion.

\section{Observations and Data}

This investigation includes previously reported astrometric and spectroscopic data, as well as new spectroscopic data taken with the W. M. Keck Observatory (WMKO). All the WMKO spectroscopic observations used for S0-2 RV measurements are summarized in Table 1.

\subsection{S0-2 Radial Velocities}

\subsubsection{Previously Reported Data}

Over the past 16 years, S0-2 has been closely monitored spectroscopically. In the published literature, $24 \mathrm{RV}$ 


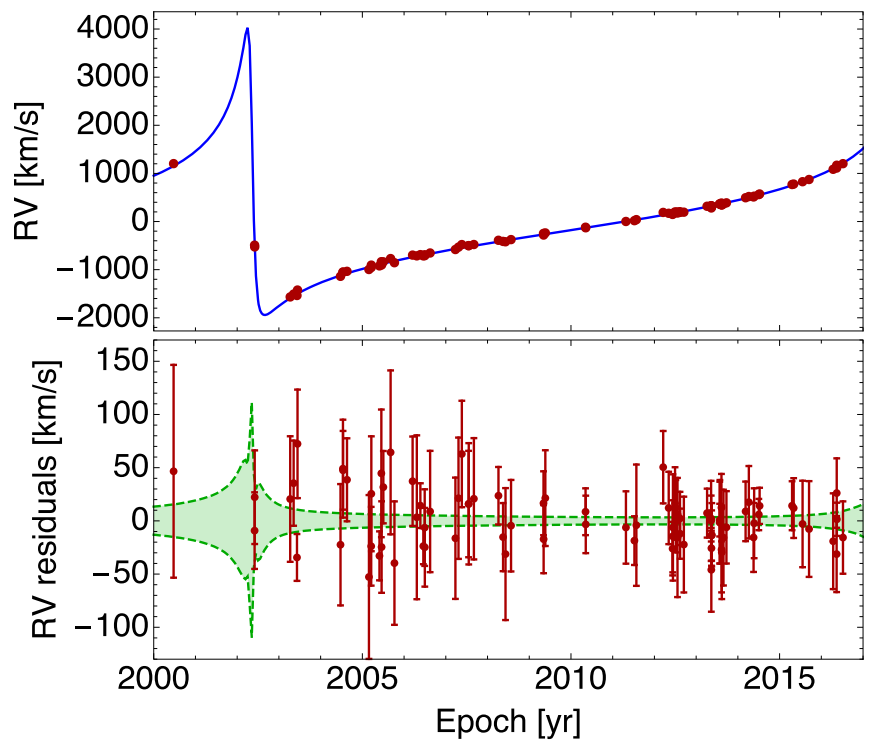

Figure 1. Top: S0-2's radial velocity measurements over time and the best-fit model. Bottom: residual radial velocity curve. The dashed lines are the $1 \sigma$ model uncertainties.

measurements, beginning in the year 2000, have been reported from WMKO (Ghez et al. 2003, 2005, 2008; Boehle et al. 2016) and 40 measurements, beginning in 2003, have been reported from the VLT (Eisenhauer et al. 2005; Gillessen et al. 2009a, 2017; Habibi et al. 2017). Many of the RV measurements are based on multiple nights of observations. For this analysis, we are interested in the presence of binaries, which for S0-2 can have periods as short as $\sim 1$ day. We therefore re-extracted $\mathrm{S} 0-2^{\prime} \mathrm{s}$ spectra from the previously calibrated WMKO data on a nightly basis for the following nights, which were previously combined: 2009 May 5 and 6 to 2009.334, 2010 May 5 and 8 to 2010.349, 2012 June 8-11 to 2012.441, 2012 July 21 and 22 to 2012.556, 2012 August 12 and 13 to 2012.616, 2013 May 14 and 16 to 2013.369, 2013 July 25-27 to 2013.566, and 2013 August 10-13 to 2013.612 (see Table 1). This increases the Keck data set to 38 points for this time period. For the VLT, 7 out of 41 epochs are reported to be derived from multiple nights of data.

\subsubsection{New Spectroscopic Data}

We report new spectroscopic observations for S0-2 obtained using the integral field spectrograph OSIRIS (Larkin et al. 2006) on the W. M. Keck I telescope with the laser guide star AO system. These data were observed between 2014 to 2016. Details about the filters and integration time related to these observations are given in Table 1. The RV observations and data analysis follow the same procedures used for earlier WMKO S0-2 RV measurements (Ghez et al. 2008; Do et al. 2013). The 12 new RV measurements, along with the RV measurements from Section 2.1.1 (34 Keck and 41 VLT) result in 87 total RV measurements, which is the sample used in this work (see Table 3).

\subsection{Characteristics of the Two Data Sets}

The Keck and VLT data sets are analyzed in a similar manner and appear to be consistent with one another. The two data sets are analyzed with the same standard spectroscopic calibration procedures and the absolute wavelength solutions are both determined from the $\mathrm{OH}$ sky emission lines. The RV of S0-2 is measured from both data sets by fitting a Gaussian to the $\mathrm{Br} \gamma$ absorption line. The reported average RV uncertainties are very similar, $33 \mathrm{~km} \mathrm{~s}^{-1}$ and $45 \mathrm{~km} \mathrm{~s}^{-1}$ for Keck and VLT, respectively. Furthermore, for the four Keck and VLT points taken within 10 days of each other, three of the points were within $1 \sigma$ of each other. The one exception is the Keck 2003.433 point, which differs from a nearby VLT point by $2 \sigma$. We conclude that there is no significant systematic difference.

\subsection{Removing SO-2's Long-term RV Variations}

Before searching for short-term RV variations, we remove the long-term RV variations from $\mathrm{S} 0-2^{\prime} \mathrm{s}$ orbital motion around the SMBH. To create the long-term RV model, a simultaneous orbital fit of S0-2 and S0-38 was performed using the same S0-2 and S0-38 astrometry and process as Boehle et al. (2016), but with the S0-2 RVs in Table 3 and S038 RVs from Boehle et al. (2016) and Gillessen et al. (2017). One additional change is the format of time used. In this work, we use Modified Julian Date (MJD). The reported time is the approximate average time of the observations taken during the night. For convenience, we also report the Universal Time (UT) and epoch time reported in Julian years of 365.25 days since J2000. Previously, Boehle et al. (2016) defined the epoch year as 365.24 days. The orbital parameters resulting from the fit are consistent with Boehle et al. (2016) within $1 \sigma$ and are presented in the Appendix A. The RV data, model, and residual are shown in Figure 1 and given in Table 3 . The average scatter around the orbit residual is $20 \mathrm{~km} \mathrm{~s}^{-1}$ with a standard deviation of $26 \mathrm{~km} \mathrm{~s}^{-1}$. The Keck and VLT data sets are individually consistent with these values.

\section{Is S0-2 a Binary?}

In this section, we use two different methods to to search for periodic signals in the RV residuals: (i) the Lomb-Scargle periodogram (Lomb 1976; Scargle 1982; Astropy Collaboration et al. 2013) and (ii) a Bayesian fit for potential binaries. The former method provides a quick overview of the data; we note that while it may not be as effective at detecting highly eccentric binaries (e.g., phase dispersion measure (Stellingwerf 1972), minimum string length (Dworetsky 1983)), it is an computationally efficient method for detecting periodic signals in unevenly spaced data (see Appendix B). The latter method provides a more complete and robust, albeit more computationally expensive approach and allows us to derive upper limits on the orbital parameters of a hypothetical binary companion to S0-2.

We can place an upper limit on the orbital period of any possible companion around S0-2 of 119.2 days based on binary disruption criteria. A binary would be tidally disrupted at closest approach to the SMBH if it has a separation greater than the Hill radius $\left(r_{\mathrm{H}}\right)$. The Hill radius is given by

$$
r_{\mathrm{H}}=a_{\mathrm{S} 0-2}\left(1-e_{\mathrm{S} 0-2}\right) \sqrt[3]{\frac{M_{\text {Primary }}}{3 M_{\mathrm{BH}}}},
$$

where $M_{\text {Primary }}$ is the primary mass, and $a_{\mathrm{S} 0-2}$ and $e_{\mathrm{S} 0-2}$ are the semimajor axis and eccentricity for the binary-black hole system (the corresponding values have been derived from the orbital fit presented in Section 2.3 whose result is presented in Appendix A). This Hill Radius limit is a conservative limit, since any eccentricity of the inner binary system would decrease the stability of the system. We take the condition 


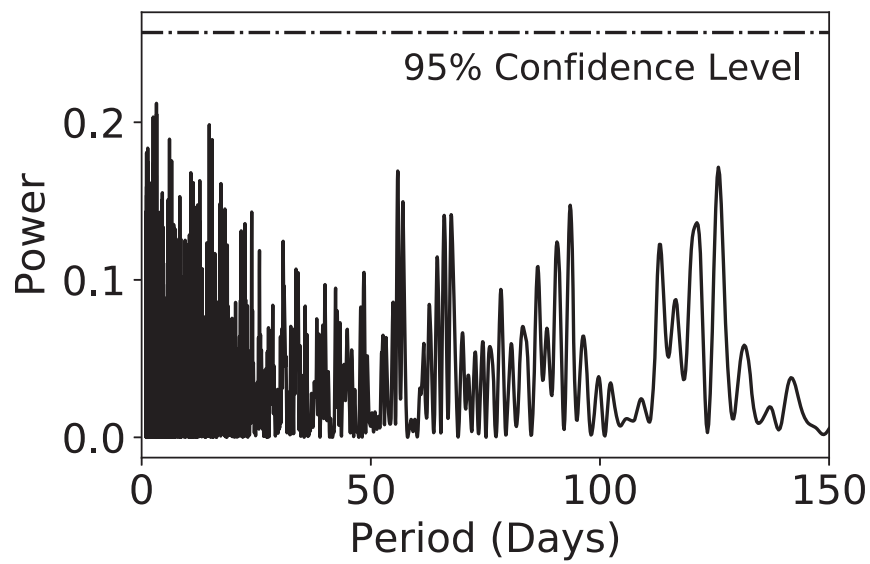

Figure 2. Lomb-Scargle periodogram of S0-2's residual RV curve. The black dashed-dotted line is the $95 \%$ confidence level detection value. No power reaches the $95 \%$ confidence level detection value, implying that no significant periodic signal is found in the observations.

$a(1+e)<r_{\mathrm{H}}$, where $e$ is the eccentricity and $a$ is the semimajor axis of the inner binary, to allow for long-term stability (Naoz 2016), which leads to the following constraint on the binary period $P$ :

$$
P^{2}<\frac{4 \pi^{2}}{3 G M_{\mathrm{BH}}} a_{\mathrm{S} 0-2}^{3} \frac{\left(1-e_{\mathrm{S} 0-2}\right)^{3}}{(1+e)^{3}} \frac{M_{\text {Primary }}}{M_{\mathrm{tot}}} .
$$

This condition needs to be fulfilled to avoid a disruption of the binary. We therefore sampled periods between 1 and 150 days to search for a significant periodic signal.

The Lomb-Scargle analysis on S0-2's RV does not reveal any statistically significant peak (see Figure 2 ). We note that the structure of this periodogram is unaffected by the model uncertainties over the period range searched. In order to determine the relationship between periodogram power and statistical significance, we ran a series of Monte Carlo simulations. We first generated 100,000 simulated residual RV curves with no periodic signal. The simulated points had the same observation times and uncertainties as the data and were drawn from a Gaussian centered around $0 \mathrm{~km} \mathrm{~s}^{-1}$. We produced a periodogram for each simulated RV curve and found the maximum peak power value. We then looked at the distribution of these maximum power values and made a cumulative distribution function (CDF). We used this CDF to determine the significance for periodic detections. These simulations set the $95 \%$ confidence level detection limit to be 0.25 , shown in a dotted line in Figure 2. The periodogram corresponding to S0-2's observations never reaches this value, which implies that no significant periodic signal is found in the current data and that observations are consistent with a single star model.

Since no evidence of a binary for S0-2 is found, we can place an upper limit on the amplitude on the RV variations induced by a binary system. In order to infer such a limit, we fit the S0-2 $\mathrm{RV}$ residuals with a binary star RV model plus a constant. The following equation was used to model the RV curve of an eccentric binary system (Hilditch 2001)

$$
\mathrm{RV}=K \frac{\sqrt{1-e^{2}} \cos E \cos \omega-\sin E \sin \omega}{1-e \cos E}
$$

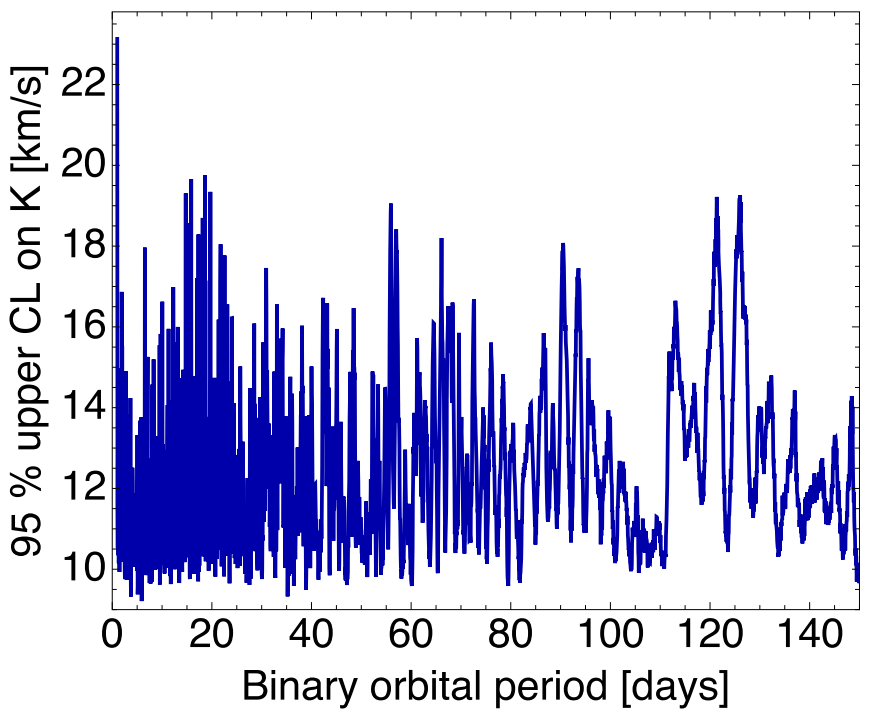

Figure 3. Upper limits on the amplitude of RV variations induced by possible undetected companion stars. Plotted at the $95 \%$ upper confidence limit are the amplitude of RV variations that could be induced by a hidden binary system ( $K$ as defined by Equations (3) and (4)) as a function of the binary orbital period.

with

$$
K=\frac{2 \pi a \sin i}{P}
$$

and where $e$ is the binary eccentricity, $\omega$ is the argument of periastron, $E$ is the eccentric anomaly determined by solving the Kepler equation, $i$ is the inclination, $P$ is the period, and $a$ is the semimajor axis. This model is parameterized using the following five variables: the offset $O$, the RV amplitude $K$, the eccentricity $e$, the argument of periastron $\omega$, and the mean longitude at $\mathbf{J} 2000$ (noted $L_{0}$ ). The use of the mean longitude at J2000 is preferred over the usual time of closest approach, which is not bounded and not defined in case of circular orbits (Hilditch 2001). For different fixed binary orbital periods $P$, we fitted this model to the RV residuals using a MultiNeST sampler (Feroz \& Hobson 2008; Feroz et al. 2009, 2013). The resulting 95\% upper confidence limit on $K$ for $\sim 3000$ orbital periods ranging from 1 to 150 days is shown in Figure 3.

Like the Lomb-Scargle analysis, this method is also sensitive to periodic signals in the data. A periodic signal would yield a significant non-zero peak in the value of $K$ in the posterior, as opposed to a power-law-decreasing posterior. This method also inherently takes into account the other orbital parameters of the binary system that can affect the shape of the curve (e.g., eccentricity).

With one further assumption, this analysis also allows us to constrain the mass of a hypothetical companion. Assuming a total mass of the system $\left(M_{\text {tot }}\right)$, we transform the sampling (i.e., the chain) resulting from the MULTINEST run into a companion mass limit using

$$
M_{\text {comp }} \sin i=\left(\frac{P M_{\mathrm{tot}}^{2}}{2 \pi G}\right)^{1 / 3} K,
$$

where $M_{\text {comp }}$ is the companion mass and $i$ is the inclination of the binary system. From this transformed chain, we can derive an upper $95 \%$ confidence limit on $M_{\text {comp }} \sin i$. This limit 


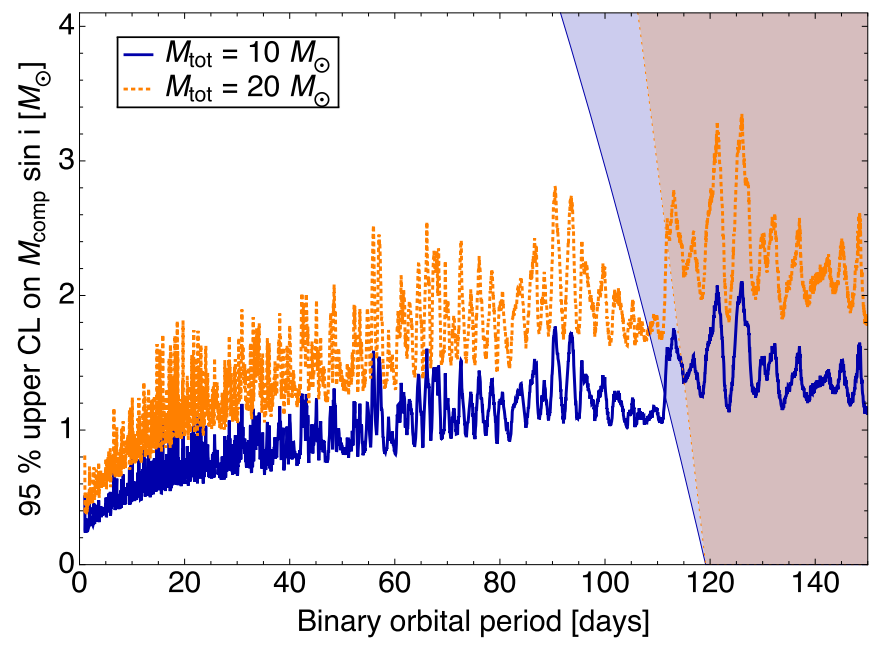

Figure 4. Upper limits on allowed companion star masses. Plotted are the $95 \%$ upper confidence limits on $M_{\text {comp }} \sin i$ as a function of the binary orbital period assuming two different values for the total mass of the binary system. The shaded regions represent the area excluded by the Hill radius limits from Equation (2), while adopting a circular orbit (which corresponds to the most conservative limit).

depends on the total mass $M_{\text {tot }}$ used in Equation (5). In this work, two extreme values for $M_{\text {tot }}$ are considered: (i) a low value of $M_{\mathrm{tot}}=10 M_{\odot}$ (Habibi et al. 2017 reported the mass of S0-2 as $13.6_{-1.8}^{+2.2} M_{\odot}$ ) and (ii) a high value of $M_{\text {tot }}=20 M_{\odot}$. The upper limit on $M_{\text {comp }} \sin i$ is shown in Figure 4, as well as the excluded region inferred by theoretical arguments based on the binary disruption criteria ${ }^{4}$ and characterized by Equation (2). The median upper $95 \%$ confidence limit for $M_{\text {comp }} \sin i$ for all periods is $1.6 M_{\odot}$ assuming a total mass of $20 M_{\odot}$ while its maximal value is $2.8 M_{\odot}$ at a 93.5-day period. These values decrease by $36 \%$ for a total mass $M_{\mathrm{tot}}$ of $10.0 M_{\odot}$.

\section{The Impact of Hidden Allowed Companions on Measurements of S0-2's Relativistic Redshift}

As anticipated by many theorists, observations of shortperiod stars orbiting the SMBH in our Galactic center are currently opening a new window into testing the gravitational theory and to measure relativistic effects (see, e.g., Rubilar \& Eckart 2001; Zucker et al. 2006; Will 2008; Borka et al. 2013; Johannsen 2016a, 2016b; Psaltis et al. 2016; Zakharov et al. 2016; Hees et al. 2017; Iorio 2017 and references therein). The relativistic redshift on S0-2's RV is the first relativistic effect expected to be detected with S0-2's closest approach in 2018 (Zucker et al. 2006; Angélil \& Saha 2010, 2011; Hees et al. 2017). This measurement of the relativistic redshift will improve in the future and will also be followed by measurements of more relativistic effects such as the advance of $\mathrm{S} 0-2$ 's periastron. In the case where $\mathrm{S} 0-2$ is a binary system, the measurement of relativistic effects like the redshift would be altered. The goal of this section is to quantify the impact of a binarity of S0-2 on the measurement of its relativistic redshift.

\footnotetext{
4 Interactions from background stars (Hopman 2009) and the eccentric KozaiLidov mechanism ( $\mathrm{Li}$ et al. 2017) can also disrupt or merge binaries. We do not consider these scenarios because these effects depend on many variables, such as the age of the binary.
}

One way to measure the relativistic redshift is to model the total $\mathrm{RV}$ as $\mathrm{RV}=[\mathrm{RV}]_{\text {Newton }}+\Upsilon[\mathrm{RV}]_{\text {rel }}$, where $[\mathrm{RV}]_{\text {Newton }}$ is the standard Newtonian RV, $\Upsilon$ is a dimensionless parameter whose value is equal to 1 in GR, and $[R V]_{\text {rel }}$ is the first-order relativistic contribution to the $\mathrm{RV}$ given by

$$
[\mathrm{RV}]_{\mathrm{rel}}=\frac{v^{2}}{2 c}+\frac{G M_{\mathrm{BH}}}{r c},
$$

where $c$ is the speed of light in a vacuum, $r$ is the norm of the star's position with respect to the $\mathrm{SMBH}$, and $v$ the norm of its velocity. The first term is a contribution due to special relativity, while the second term corresponds to the gravitational redshift. For a Keplerian orbit, the two contributions are exactly the same (up to a constant factor), meaning that only their combination can be measured. The relativistic redshift contribution to $\mathrm{S} 0-2^{\prime} \mathrm{s} \mathrm{RV}$ reaches $200 \mathrm{~km} \mathrm{~s}^{-1}$ at closest approach in 2018, while the Newtonian part ranges from -2000 to $4000 \mathrm{~km} \mathrm{~s}^{-1}$ (see Figure 1). The idea is to fit $\Upsilon$ simultaneously with the other parameters in the orbital fit: a value significantly different from 0 but compatible with 1 would be a successful detection of the relativistic redshift, while a value significantly different from 1 would indicate a deviation from GR. The goal of this section is to quantify the impact of a plausible binary for S02 on the determination of $\Upsilon$.

The methodology consists of simulating data assuming S0-2 is a binary star using a relativistic modeling (in particular we use $\Upsilon=1$ ) and analyze these data using a modeling where S02 is a single star and where $\Upsilon$ is a free parameter. The deviation $\Upsilon-1$ obtained in this analysis is therefore entirely due to the fact that S0-2 has been simulated as a binary star.

More precisely, we simulate astrometric and RV data for S0-2 using a relativistic modeling that includes the Römer time delay and the redshift (see, e.g., Alexander 2005). The simulated epochs correspond to epochs where we actually have data (see Table 3) and for all simulated data, we assign an uncertainty that corresponds to the actual measurement. In addition to existing data, we included simulated data for 2018: 10 spectroscopic observations that were assigned an uncertainty of $25 \mathrm{~km} \mathrm{~s}^{-1}$ and four astrometric observations that were assigned an uncertainty of 0.3 mas. The epochs for these additional observations have been chosen to optimize a redshift measurement within the 2018 observation window. At this step, the simulated data corresponds to perfect measurements in the case where S0-2 is a single star. Therefore, an orbital fit using these simulated data recovers the input value, i.e., it gives an estimate of $\Upsilon=1$ as expected.

To these simulated data, we then add the signature produced by a binary star given by Equation (3). The obtained data now correspond to a binary system. This data set is then used in a one-star orbital fit that includes the $G M_{\mathrm{BH}}$, the distance to our Galactic center $R_{0}$, the position and velocity of the $\mathrm{BH}$, the six orbital parameters for the star, and the relativistic redshift parameter $\Upsilon$. The impact of the binarity of S0-2 on the redshift measurement will be given by the estimated value ${ }^{5}$ of $\Upsilon-1$.

This procedure has been performed for six different binary orbital periods: 2, 5, 10, 25, 50, and 100 days. For each of these periods, we draw 2000 samples for the other binary orbital parameters (eccentricity $e, \mathrm{RV}$ amplitude $K$, argument of

\footnotetext{
We use the median as the estimated value from the MultiNeST fit.
} 

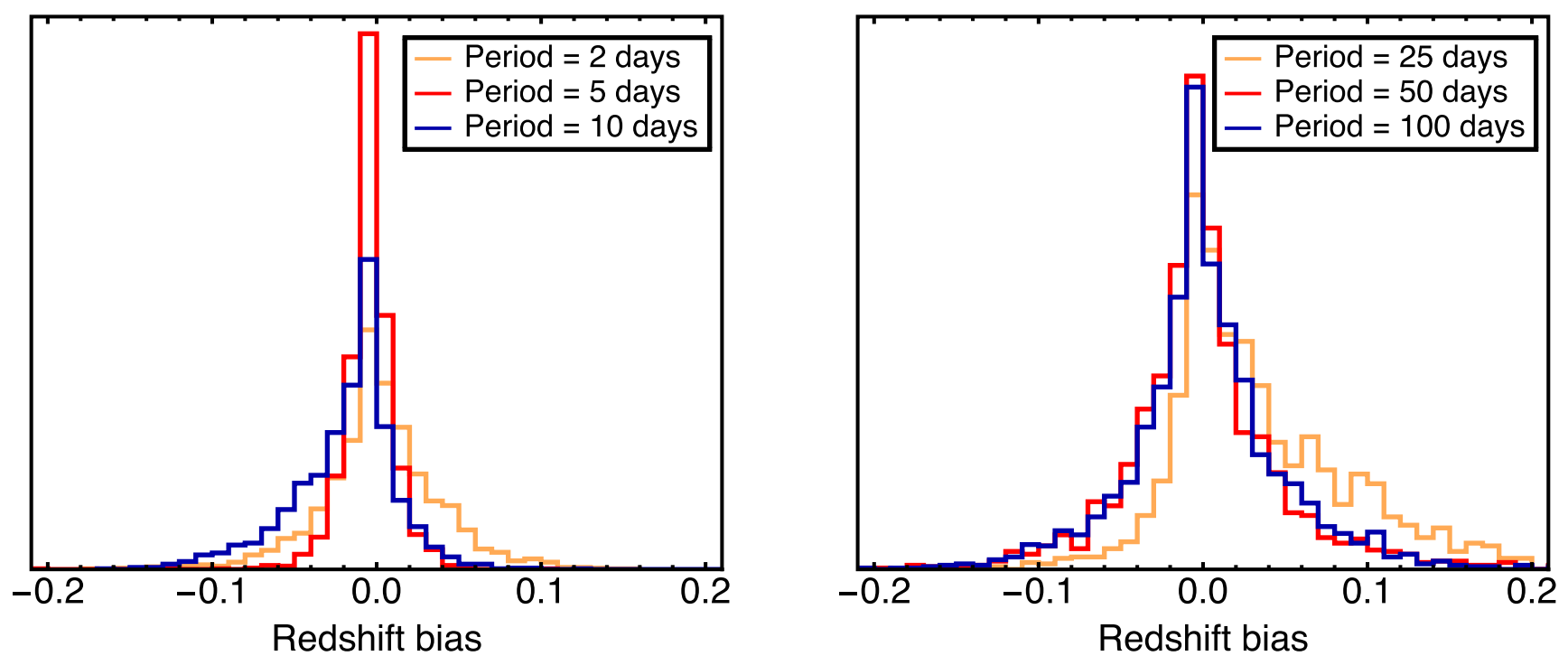

Figure 5. Bias on the estimation of relativistic redshift $(\Upsilon-1)$. Plotted are the imposed biases from model fits using simulated S0-2 observations, which extend through 2018 and include RV variations induced by the binary star systems allowed by the current S0-2 RV data. The different curves correspond to different binary periods. In general, the periods that have stricter companion limits result in smaller biases; the median binary amplitude limits correspond to a redshift bias of 0.03 .

periastron $\omega$, and longitude at $\mathrm{J} 2000 L_{0}$ ) from the posterior probability distribution function of the fit described in Section 3. The resulting distributions for the bias in the redshift measurement $\Upsilon-1$ are presented in Figure 5. Furthermore, the values of the half of the $68 \%$ upper limit on the absolute value of the redshift bias are given in Table 2 . In general, the periods that have stricter limits on the amplitude of the RV variations $(K)$ induced by a hidden binary result in smaller biases; the median binary amplitude limits correspond to a redshift bias of 0.03 .

Although a plausible binary for S0-2 can bias the measurement of the relativistic redshift, this bias is always smaller than the uncertainty corresponding to a $5 \sigma$ detection of the redshift (a $5 \sigma$ detection is characterized by $\sigma_{\Upsilon}=0.2$ ).

\section{Discussion and Conclusion}

\subsection{SO-2 in Its Astrophysical Context}

This is the first work to investigate S0-2 as a spectroscopic binary. Previous searches have concentrated on brighter sources such as IRS16SW and E60 (Pfuhl et al. 2014), located beyond the $\mathrm{S}$-star cluster region (outside of $\sim 0.04 \mathrm{pc})$. This work has pushed the RV searches to 2 magnitudes fainter from $K=12.0$ (E60) down to $K=14.0$; physically, this magnitude difference corresponds to the difference between evolved Wolf-Rayet stars and mainsequence B stars. The improvements here are driven by the large number of RV measurements available for S0-2 from the long-baseline monitoring programs for this source (e.g., Boehle et al. 2016; Gillessen et al. 2017).

While we detect no significant binary signal in the RV variations, we have been able to place stringent limits on the companion mass. Our limits of $1.6 M_{\odot}$ are consistent with other observations of the star. For example, given our $95 \%$ confidence limit of $1.6 M_{\odot}$, a star would have an observed brightness of $K \sim 18 \mathrm{mag}$ at the Galactic center, corresponding to a factor of 40 times less flux than S0-2. This brightness ratio is consistent with the fact that $\mathrm{S} 0-2^{\prime} \mathrm{s}$ spectrum shows no sign of another set of spectral features, even with 10 years of spectra combined (Habibi et al. 2017). While our
Table 2

Impact of a Binarity of S0-2 on a Measurement of the Relativistic Redshift

\begin{tabular}{lcc}
\hline \hline Binary Period & $\begin{array}{c}95 \% \text { C.L. on } \\
K\left(\mathrm{~km} \mathrm{~s}^{-1}\right)^{\mathrm{a}}\end{array}$ & $\begin{array}{c}\text { Uncertainty on the Redshift } \\
\text { due to the Binarity }\left(\sigma_{\Upsilon}\right)\end{array}$ \\
\hline 2 days & 16.0 & 0.031 \\
5 days & 10.4 & 0.011 \\
10 days & 12.2 & 0.026 \\
25 days & 12.6 & 0.051 \\
50 days & 11.0 & 0.036 \\
100 days & 12.9 & 0.039 \\
\hline
\end{tabular}

Note.

${ }^{\text {a }} K$ is the amplitude of the RV variations induced by a binary as introduced in Equations (3) and (4). The 95\% upper confidence limits on $K$ are presented in Figure 3.

mass limits have a sin $i$ degeneracy, the lack of detection of a double-lined source also shows that a face-on binary system is also very unlikely.

The lack of a binary companion does not distinguish different formation scenarios for $\mathrm{S} 0-2$ at this time. No companion is expected if $\mathrm{S} 0-2$ is the remaining companion of a hypervelocity star (e.g., Hills 1988; Yu \& Tremaine 2003; Perets et al. 2009), or if it is the product of a merger (e.g., Sana et al. 2012; Phifer et al. 2013; Witzel et al. 2014; Stephan et al. 2016). Scattering from the young star cluster (at $0.1-0.5 \mathrm{pc}$ ) could also bring S0-2 in without a companion (Perets et al. 2007; Madigan et al. 2014). While the current observations of S0-2 are unable to differentiate between these scenarios, companion searches of the other S-stars should be able to provide a much more comprehensive test of the formation scenarios for the S-stars. We have concentrated on S0-2 because the other S-stars are all fainter, which results in lower precision in their RV compared to S0-2. Additional measurements should improve the sensitivity of companion searches.

\subsection{SO-2 as a New Probe of Fundamental Physics}

The relativistic redshift at S0-2's closest approach in 2018 will be the first measurement of its kind, so understanding all sources of bias will be especially important for a significant detection. 
Table 3

S0-2 Radial Velocity Measurements

\begin{tabular}{|c|c|c|c|c|c|c|c|c|c|c|}
\hline UT & MJD & Epoch $^{\mathrm{a}}$ & $\underset{\left(\mathrm{km} \mathrm{s}^{-1}\right)}{\mathrm{RV}_{\text {obs }}}$ & $\begin{array}{c}\mathrm{RV} \sigma \\
\left(\mathrm{km} \mathrm{s}^{-1}\right)\end{array}$ & $\begin{array}{c}V_{\mathrm{LSR}}{ }^{\mathrm{b}} \\
\left(\mathrm{km} \mathrm{s}^{-1}\right)\end{array}$ & $\begin{array}{c}\mathrm{RV}_{\mathrm{LSR}}{ }^{\mathrm{c}} \\
\left(\mathrm{km} \mathrm{s}^{-1}\right)\end{array}$ & $\begin{array}{c}\mathrm{RV}^{\mathrm{d}} \\
\text { Source }\end{array}$ & $\begin{array}{c}\text { Model } \\
\left(\mathrm{km} \mathrm{s}^{-1}\right)\end{array}$ & $\begin{array}{l}\text { Model } \sigma \\
\left(\mathrm{km} \mathrm{s}^{-1}\right)\end{array}$ & $\begin{array}{c}\text { Residual }^{\mathrm{e}} \\
\left(\mathrm{km} \mathrm{s}^{-1}\right)\end{array}$ \\
\hline 2000 Jun 23 & 51718.50 & 2000.476 & 1192 & 100 & 7 & 1199 & (2) & 1152 & 15 & 47 \\
\hline 2002 Jun 02 & 52427.50 & 2002.418 & -513 & 36 & 18 & -495 & (2) & -486 & 28 & -9 \\
\hline 2002 Jun 03 & 52428.50 & 2002.420 & -550 & 44 & 18 & -532 & (2) & -554 & 27 & 22 \\
\hline 2003 Apr 09 & 52739.23 & 2003.271 & $\ldots$ & 59 & $\ldots$ & -1571 & (3) & -1592 & 12 & 21 \\
\hline 2003 May 09 & 52769.18 & 2003.353 & $\ldots$ & 40 & $\cdots$ & -1512 & (3) & -1547 & 11 & 35 \\
\hline 2003 Jun 08 & 52798.50 & 2003.433 & -1556 & 22 & 15 & -1541 & (2) & -1507 & 10 & -34 \\
\hline 2003 Jun 12 & 52803.15 & 2003.446 & $\ldots$ & 51 & $\ldots$ & -1428 & (3) & -1500 & 10 & 72 \\
\hline 2004 Jun 23 & 53179.50 & 2004.476 & -1151 & 57 & 8 & -1143 & (2) & -1121 & 7 & -22 \\
\hline 2004 Jul 14 & 53200.91 & 2004.535 & $\ldots$ & 46 & $\ldots$ & -1055 & (3) & -1104 & 7 & 49 \\
\hline 2004 Jul 15 & 53201.64 & 2004.537 & $\ldots$ & 37 & $\ldots$ & -1056 & (3) & -1104 & 7 & 48 \\
\hline 2004 Aug 18 & 53236.34 & $2004.632^{\mathrm{f}}$ & $\ldots$ & 39 & $\ldots$ & -1039 & (3) & -1078 & 7 & 39 \\
\hline $2005-02-26$ & 53428.46 & 2005.158 & $\ldots$ & 77 & $\ldots$ & -1001 & (3) & -948 & 6 & -53 \\
\hline 2005 Mar 18 & 53448.18 & 2005.212 & $\ldots$ & 37 & $\ldots$ & -960 & (3) & -936 & 6 & -24 \\
\hline 2005 Mar 19 & 53449.28 & 2005.215 & $\ldots$ & 54 & $\ldots$ & -910 & (3) & -935 & 6 & 25 \\
\hline 2005 May 30 & 53520.50 & 2005.410 & -945 & 23 & 19 & -926 & (2) & -893 & 6 & -33 \\
\hline 2005 Jun 15 & 53536.94 & 2005.455 & $\ldots$ & 60 & $\ldots$ & -839 & (3) & -884 & 6 & 45 \\
\hline 2005 Jun 17 & 53539.13 & 2005.461 & $\ldots$ & 43 & $\ldots$ & -907 & (3) & -882 & 6 & -25 \\
\hline $2005 \mathrm{Jul} 03$ & 53554.50 & 2005.503 & -845 & 34 & 3 & -842 & (2) & -874 & 6 & 32 \\
\hline 2005 Sep 04 & 53618.02 & $2005.677^{\mathrm{f}}$ & $\ldots$ & 77 & $\ldots$ & -774 & (3) & -838 & 6 & 64 \\
\hline 2005 Oct 08 & 53651.63 & $2005.769^{f}$ & $\ldots$ & 58 & $\ldots$ & -860 & (3) & -820 & 6 & -40 \\
\hline 2006 Mar 15 & 53810.51 & 2006.204 & $\ldots$ & 42 & $\ldots$ & -702 & (3) & -739 & 5 & 37 \\
\hline 2006 Apr 21 & 53847.40 & 2006.305 & $\ldots$ & 77 & $\ldots$ & -718 & (3) & -721 & 5 & 3 \\
\hline 2006 May 23 & 53878.50 & 2006.390 & -715 & 21 & 23 & -692 & (2) & -707 & 5 & 14 \\
\hline 2006 Jun 18 & 53904.50 & 2006.461 & -728 & 17 & 10 & -718 & (2) & -694 & 5 & -24 \\
\hline 2006 Jun 30 & 53916.50 & 2006.494 & -699 & 36 & 4 & -695 & (2) & -689 & 5 & -6 \\
\hline $2006 \mathrm{Jul} 01$ & 53917.50 & 2006.497 & -717 & 37 & 4 & -713 & (2) & -688 & 5 & -25 \\
\hline 2006 Aug 16 & 53963.92 & $2006.624^{\mathrm{f}}$ & $\ldots$ & 57 & $\ldots$ & -658 & (3) & -667 & 5 & 9 \\
\hline 2007 Mar 25 & 54185.26 & 2007.230 & $\ldots$ & 57 & $\ldots$ & -586 & (3) & -570 & 5 & -16 \\
\hline 2007 Apr 21 & 54212.29 & 2007.304 & $\ldots$ & 57 & $\ldots$ & -537 & (3) & -558 & 5 & 21 \\
\hline 2007 May 21 & 54241.50 & 2007.384 & -507 & 50 & 24 & -483 & (2) & -546 & 5 & 63 \\
\hline 2007 Jul 19 & 54300.29 & 2007.545 & -502 & 50 & -4 & -506 & (2) & -522 & 5 & 16 \\
\hline 2007 Jul 20 & 54302.14 & $2007.550^{\mathrm{f}}$ & $\ldots$ & 57 & $\ldots$ & -505 & (3) & -521 & 5 & 16 \\
\hline 2007 Sep 03 & 54347.06 & $2007.673^{\mathrm{f}}$ & $\ldots$ & 57 & $\ldots$ & -482 & (3) & -503 & 4 & 21 \\
\hline 2008 Apr 05 & 54562.20 & $2008.262^{f}$ & $\ldots$ & 27 & $\ldots$ & -394 & (3) & -418 & 4 & 24 \\
\hline 2008 May 16 & 54602.50 & 2008.372 & -443 & 32 & 26 & -417 & (2) & -402 & 4 & -15 \\
\hline 2008 Jun 06 & 54623.92 & 2008.431 & $\ldots$ & 62 & $\ldots$ & -425 & (3) & -394 & 4 & -31 \\
\hline 2008 Jul 25 & 54672.28 & 2008.563 & -373 & 43 & -7 & -380 & (2) & -375 & 4 & -5 \\
\hline 2009 May 05 & 54956.50 & 2009.342 & -282 & 30 & 30 & -252 & (2) & -268 & 4 & 17 \\
\hline 2009 May 06 & 54957.50 & 2009.344 & -315 & 32 & 30 & -285 & (2) & -268 & 4 & -17 \\
\hline 2009 May 20 & 54972.37 & 2009.385 & $\ldots$ & 45 & $\ldots$ & -241 & (3) & -262 & 4 & 21 \\
\hline 2010 May 08 & 55324.50 & 2010.349 & -152 & 22 & 29 & -123 & (2) & -131 & 4 & 9 \\
\hline 2010 May 09 & 55326.30 & 2010.354 & $\ldots$ & 27 & $\ldots$ & -134 & (3) & -131 & 4 & -3 \\
\hline 2011 Apr 26 & 55678.03 & 2011.317 & $\ldots$ & 34 & $\ldots$ & -3 & (3) & 3 & 3 & -6 \\
\hline $2011 \mathrm{Jul} 10$ & 55752.33 & 2011.520 & 14 & 23 & 0 & 14 & (2) & 32 & 3 & -19 \\
\hline 2011 Jul 26 & 55769.35 & 2011.567 & $\ldots$ & 57 & $\ldots$ & 35 & (3) & 39 & 3 & -4 \\
\hline 2012 Mar 17 & 56004.20 & 2012.210 & $\ldots$ & 34 & $\ldots$ & 185 & (3) & 135 & 3 & 50 \\
\hline 2012 May 04 & 56052.42 & 2012.342 & $\ldots$ & 34 & $\ldots$ & 167 & (3) & 155 & 3 & 12 \\
\hline 2012 Jun 08 & 56086.50 & 2012.435 & 128 & 25 & 15 & 143 & (2) & 169 & 3 & -26 \\
\hline 2012 Jun 09 & 56087.46 & 2012.438 & 141 & 34 & 14 & 155 & (2) & 170 & 3 & -15 \\
\hline 2012 Jun 11 & 56089.50 & 2012.444 & 151 & 50 & 13 & 164 & (2) & 171 & 3 & -6 \\
\hline 2012 Jun 29 & 56107.93 & 2012.494 & $\ldots$ & 34 & $\ldots$ & 195 & (3) & 179 & 3 & 16 \\
\hline 2012 Jul 06 & 56114.87 & 2012.513 & $\ldots$ & 34 & $\ldots$ & 186 & (3) & 182 & 3 & 4 \\
\hline 2012 Jul 21 & 56129.31 & 2012.553 & 178 & 56 & -6 & 172 & (2) & 188 & 3 & -16 \\
\hline 2012 Jul 22 & 56130.31 & 2012.555 & 200 & 8 & -6 & 194 & (2) & 188 & 3 & 6 \\
\hline 2012 Aug 12 & 56151.33 & 2012.613 & 213 & 25 & -13 & 200 & (2) & 197 & 3 & 2 \\
\hline 2012 Aug 13 & 56152.27 & 2012.615 & 199 & 24 & -14 & 186 & (2) & 198 & 3 & -12 \\
\hline 2012 Sep 14 & 56185.00 & 2012.705 & $\ldots$ & 45 & $\ldots$ & 190 & (3) & 212 & 3 & -22 \\
\hline 2013 Apr 05 & 56388.45 & 2013.262 & $\ldots$ & 23 & $\ldots$ & 313 & (3) & 306 & 3 & 7 \\
\hline 2013 May 11 & 56423.50 & 2013.358 & 295 & 38 & 28 & 323 & (1) & 323 & 3 & -0 \\
\hline 2013 May 12 & 56424.50 & 2013.361 & 298 & 22 & 27 & 325 & (1) & 323 & 3 & 2 \\
\hline 2013 May 13 & 56425.50 & 2013.363 & 251 & 39 & 27 & 278 & (1) & 324 & 3 & -46 \\
\hline 2013 May 14 & 56426.50 & 2013.366 & 272 & 16 & 27 & 298 & (2) & 324 & 3 & -26 \\
\hline 2013 May 16 & 56428.50 & 2013.372 & 286 & 24 & 26 & 311 & (2) & 325 & 3 & -14 \\
\hline
\end{tabular}


Table 3

(Continued)

\begin{tabular}{|c|c|c|c|c|c|c|c|c|c|c|}
\hline UT & MJD & Epoch $^{\mathrm{a}}$ & $\underset{\left(\mathrm{km} \mathrm{s}^{-1}\right)}{\mathrm{RV}_{\mathrm{obs}_{1}}}$ & $\begin{array}{c}\mathrm{RV} \mathrm{\sigma} \\
\left(\mathrm{km} \mathrm{s}^{-1}\right)\end{array}$ & $\begin{array}{c}V_{\mathrm{LSR}}{ }^{\mathrm{b}} \\
\left(\mathrm{km} \mathrm{s}^{-1}\right)\end{array}$ & $\begin{array}{c}\mathrm{RV}_{\mathrm{LSR}}{ }^{\mathrm{c}} \\
\left(\mathrm{km} \mathrm{s}^{-1}\right)\end{array}$ & $\begin{array}{c}\mathrm{RV}^{\mathrm{d}} \\
\text { Source }\end{array}$ & $\begin{array}{c}\text { Model } \\
\left(\mathrm{km} \mathrm{s}^{-1}\right)\end{array}$ & $\begin{array}{l}\text { Model } \sigma \\
\left(\mathrm{km} \mathrm{s}^{-1}\right)\end{array}$ & $\begin{array}{l}\text { Residual }^{\mathrm{e}} \\
\left(\mathrm{km} \mathrm{s}^{-1}\right)\end{array}$ \\
\hline 2013 May 17 & 56429.50 & 2013.374 & 287 & 30 & 25 & 312 & (1) & 326 & 3 & -13 \\
\hline 2013 Jul 25 & 56498.33 & 2013.563 & 367 & 15 & -7 & 360 & (2) & 360 & 4 & 1 \\
\hline 2013 Jul 26 & 56499.34 & 2013.566 & 366 & 39 & -7 & 359 & (2) & 360 & 4 & -2 \\
\hline 2013 Jul 27 & 56500.33 & 2013.568 & 367 & 39 & -8 & 360 & (2) & 361 & 4 & -1 \\
\hline 2013 Aug 10 & 56514.29 & 2013.607 & 393 & 32 & -13 & 380 & (2) & 368 & 4 & 12 \\
\hline 2013 Aug 11 & 56515.31 & 2013.609 & 354 & 40 & -13 & 341 & (2) & 368 & 4 & -27 \\
\hline 2013 Aug 13 & 56517.29 & 2013.615 & 353 & 44 & -14 & 340 & (2) & 369 & 4 & -30 \\
\hline 2013 Aug 27 & 56531.99 & 2013.655 & $\ldots$ & 45 & $\ldots$ & 361 & (3) & 377 & 4 & -16 \\
\hline 2013 Sep 22 & 56557.92 & 2013.726 & $\ldots$ & 34 & $\ldots$ & 384 & (3) & 390 & 4 & -6 \\
\hline 2014 Mar 08 & 56725.57 & 2014.185 & $\ldots$ & 28 & $\ldots$ & 490 & (3) & 481 & 4 & 9 \\
\hline 2014 Apr 06 & 56754.06 & 2014.263 & $\ldots$ & 34 & $\ldots$ & 515 & (3) & 497 & 4 & 18 \\
\hline 2014 May 17 & 56795.50 & 2014.376 & 481 & 32 & 25 & 506 & (1) & 522 & 4 & -16 \\
\hline 2014 May 22 & 56800.50 & 2014.390 & 500 & 33 & 23 & 523 & (1) & 525 & 4 & -2 \\
\hline 2014 Jul 02 & 56841.36 & 2014.502 & 553 & 15 & 3 & 556 & (1) & 550 & 4 & 6 \\
\hline 2014 Jul 09 & 56848.30 & 2014.521 & $\ldots$ & 17 & $\ldots$ & 568 & (3) & 554 & 4 & 14 \\
\hline 2015 Apr 19 & 57132.46 & 2015.299 & $\cdots$ & 23 & $\cdots$ & 765 & (3) & 751 & 5 & 14 \\
\hline 2015 May 03 & 57146.50 & 2015.337 & 743 & 28 & 31 & 774 & (1) & 762 & 5 & 12 \\
\hline 2015 Jul 20 & 57224.35 & 2015.551 & 829 & 41 & -5 & 823 & (1) & 826 & 5 & -3 \\
\hline 2015 Sep 15 & 57281.12 & 2015.706 & $\ldots$ & 45 & $\ldots$ & 869 & (3) & 877 & 5 & -8 \\
\hline 2016 Apr 13 & 57492.23 & 2016.284 & $\ldots$ & 45 & $\ldots$ & 1081 & (3) & 1100 & 8 & -19 \\
\hline 2016 May 14 & 57522.50 & 2016.367 & 1081 & 36 & 26 & 1107 & (1) & 1138 & 8 & -31 \\
\hline 2016 May 15 & 57523.50 & 2016.370 & 1139 & 33 & 26 & 1165 & (1) & 1140 & 8 & 26 \\
\hline 2016 May 16 & 57524.50 & 2016.372 & 1117 & 16 & 26 & 1142 & (1) & 1141 & 8 & 1 \\
\hline 2016 Jul 08 & 57578.06 & 2016.519 & $\ldots$ & 34 & $\cdots$ & 1198 & (3) & 1214 & 9 & -16 \\
\hline
\end{tabular}

Notes.

${ }^{\mathrm{a}}$ The epoch time is reported in Julian years as 365.25 days since J2000.

$\mathrm{b}$ The values came from rvcorrect task in IRAF, with an error of less than $1 \mathrm{~km} \mathrm{~s}^{-1}$ (Kerr \& Lynden-Bell 1986). For ease of viewing, values have been rounded.

${ }^{\mathrm{c}}$ Velocity after applying the $V_{\text {LSR }}$ correction. For ease of viewing, values have been rounded.

${ }^{\mathrm{d}}$ Measurements reported in (1) this work, (2) Boehle et al. (2016), and (3) Gillessen et al. (2017).

e $\mathrm{RV}_{\mathrm{LSR}}$-model. For ease of viewing, values have been rounded.

${ }^{\mathrm{f}}$ VLT combined night data.

(This table is available in machine-readable form.)

We have shown that a binary companion below our current detection limit for S0-2 can bias this measurement, as shown in Section 4. Nevertheless, this bias is always smaller than the uncertainty corresponding to a $5 \sigma$ detection of the redshift. The values reported in Table 2 should be taken into account in the estimation of the uncertainty produced by all systematic effects in 2018. We would like to emphasize that the 2018 observation campaign is expected to reduce this possible bias.

Continued monitoring of S0-2 beyond 2018 provides further opportunities to observe other relativistic effects, such as the advance of the periastron. The impact that a plausible binary system would have on these relativistic measurements is left for future work.

We are grateful for the helpful and constructive comments from the referee. We thank M. R. Morris and E. E. Becklin for their comments and long-term efforts on the Galactic Center Orbits Initiative. We also thank the staff of the Keck Observatory, especially Jim Lyke, Randy Campbell, Gary Puniwai, Heather Hershey, Hien Tran, Scott Dahm, Jason Mcllroy, Joel Hicock, and Terry Stickel, for all their help in obtaining the new observations. Support for this work at UCLA was provided by NSF grant AST-1412615, the W. M. Keck Foundation for support of the NStarsOrbits Project, the LevineLeichtman Family Foundation, Ken and Eileen Kaplan Student
Support Fund, the Preston Family Graduate Fellowship (held by D.C. and A.G.), the Galactic Center Board of Advisors, and the Janet Marott Student Travel Awards. The W. M. Keck Observatory is operated as a scientific partnership among the California Institute of Technology, the University of California, and the National Aeronautics and Space Administration. The authors wish to recognize that the summit of Maunakea has always held a very significant cultural role for the indigenous Hawaiian community. We are most fortunate to have the opportunity to observe from this mountain. The Observatory was made possible by the generous financial support of the W. M. Keck Foundation.

\section{Appendix A Orbital Fit}

The model for S0-2's long-term RV variation is based on a joint orbital fit of S0-2 and S0-38. We used the same S0-2 and S0-38 astrometry ${ }^{6}$ and process as Boehle et al. (2016), S0-38 RV from Gillessen et al. (2017), but with the S0-2 RVs from Table 3. It should also be noted that the impact of $\mathrm{S} 0-38$ is negligible for this S0-2 binary study. The resulting orbital

\footnotetext{
6 We do not report new astrometric measurements, as additional astrometric data are not expected to significantly affect S0-2's RV curve.
} 

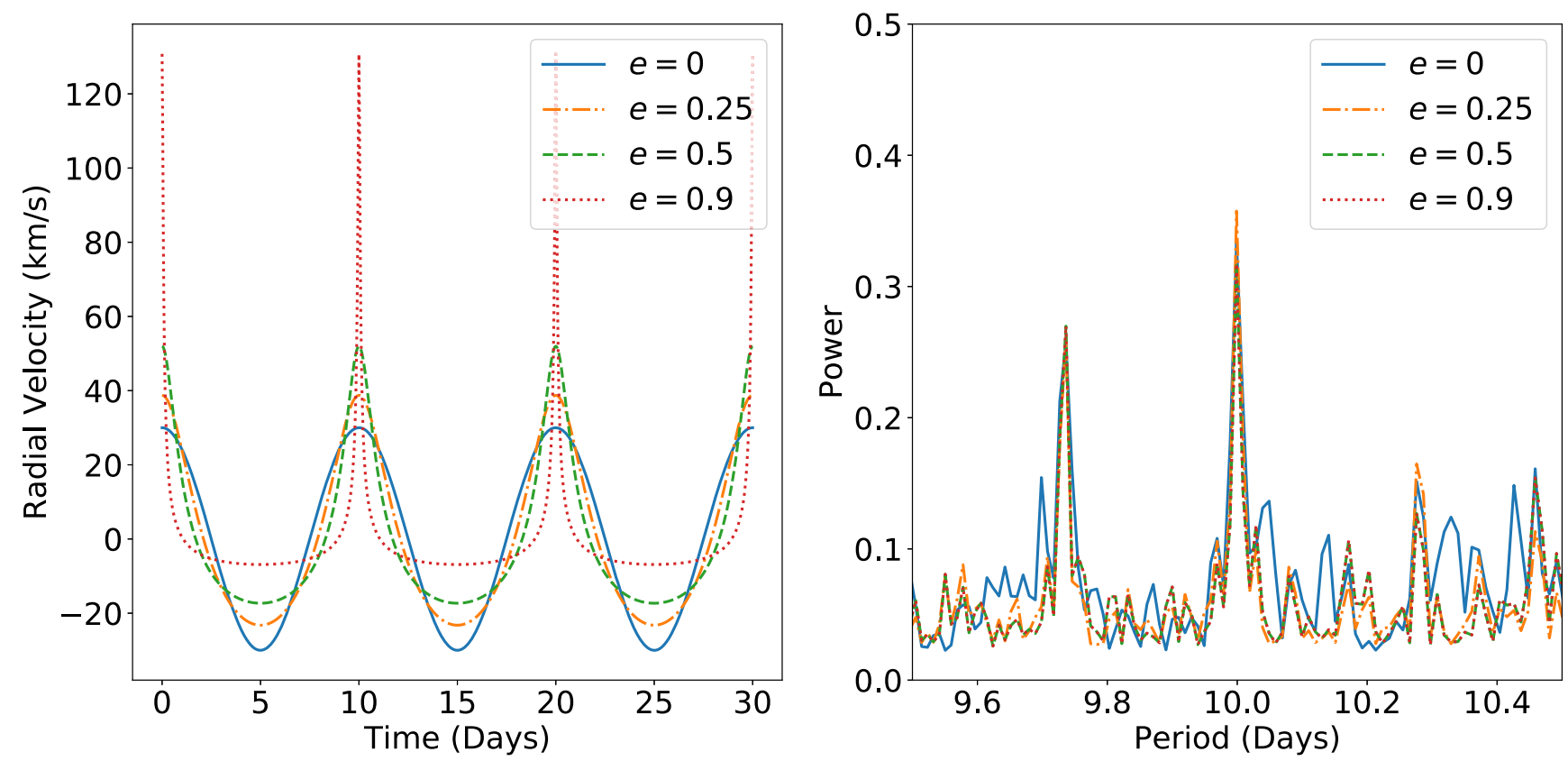

Figure 6. Left: sample radial velocity curves with different eccentricities. Each curve has a period of 10 days, a $K$ value of $30 \mathrm{~km} \mathrm{~s}^{-1}$, and a $\omega$ of $0^{\circ}$. Each curve was sampled at the same times as our data. Right: median Lomb-Scargle power values for each set of 100,000 simulations run for the different eccentric curves.

Table 4

Results from Orbital Fit

\begin{tabular}{|c|c|}
\hline Model Parameter (units) & Parameter Value $^{\mathrm{a}}$ \\
\hline \multicolumn{2}{|l|}{ Black hole Properties: } \\
\hline Distance $(\mathrm{kpc})$ & $7.93 \pm 0.13 \pm 0.04$ \\
\hline Mass $\left(10^{6} M_{\odot}\right)$ & $4.03 \pm 0.14 \pm 0.04$ \\
\hline$X$ position of Sgr A* (mas) & $2.17 \pm 0.47 \pm 1.90$ \\
\hline$Y$ position of $\mathrm{Sgr} \mathrm{A}^{*}$ (mas) & $-4.31 \pm 0.60 \pm 1.23$ \\
\hline$X$ velocity $\left({\left.\operatorname{mas~} \mathrm{yr}^{-1}\right)}^{\prime}\right.$ & $-0.11 \pm 0.03 \pm 0.13$ \\
\hline$Y$ velocity $\left(\right.$ mas $\mathrm{yr}^{-1}$ ) & $0.67 \pm 0.06 \pm 0.22$ \\
\hline$Z$ velocity $\left(\mathrm{km} \mathrm{s}^{-1}\right)$ & $-9.99 \pm 6.25 \pm 4.28$ \\
\hline \multicolumn{2}{|l|}{ S0-2 Properties: } \\
\hline Period (year) & $15.92 \pm 0.04$ \\
\hline Time of closest approach (year) & $2018.266 \pm 0.04$ \\
\hline Eccentricity & $0.892 \pm 0.002$ \\
\hline Inclination (deg) & $134.3 \pm 0.3$ \\
\hline Argument of periapse (deg) & $66.7 \pm 0.5$ \\
\hline Angle of the ascending node (deg) & $228.0 \pm 0.5$ \\
\hline \multicolumn{2}{|l|}{ S0-38 Properties: } \\
\hline Period (year) & $19.20 \pm 0.2$ \\
\hline Time of closest approach (year) & $2003.1 \pm 0.04$ \\
\hline Eccentricity & $0.811 \pm 0.004$ \\
\hline Inclination (deg) & $170 \pm 2$ \\
\hline Argument of periapse (deg) & $194 \pm 160$ \\
\hline Angle of the ascending node (deg) & $79 \pm 24$ \\
\hline
\end{tabular}

Note.

${ }^{\text {a }}$ The first error term for each best-fit value corresponds to the statistical error determined by the orbital fit. For the black hole parameters, the second error term corresponds to jackknife uncertainties from the reference frame, which were reported in Boehle et al. (2016). parameters are listed in Table 4, with all results being consistent with Boehle et al. (2016) within $1 \sigma$.

\section{Appendix B Lomb-Scargle Method}

In this work, we used the Lomb-Scargle method to look for periodic signals in the S0-2 data. The Lomb-Scargle method works best at detecting sinusoidal signals, which correspond to a circular binary system. However, as binaries become eccentric, their RV curve deviates more from a perfect sine wave. Although their curves are periodic, their non-sinusoidal shapes could lead to reduced sensitivity using the Lomb-Scargle periodogram.

We explored the method's sensitivity to eccentricity by generating four sets of 100,000 simulated eccentric binary RV curves (see Equation (3)). The first set of curves had $e=0$, the second set had $e=0.25$, the third set had $e=0.5$, and the fourth set had $e=0.9$. All curves had the same period of 10 days, a $K$ value of $30 \mathrm{~km} \mathrm{~s}^{-1}$, and a $\omega$ of $0^{\circ}$ (Figure 6). These curves were also sampled at the same times as our data. We ran each set of curves through the Lomb-Scargle analysis and took the median power values for each period. The LombScargle method successfully identified the 10-day period in the different sets of simulated curves. The median power values at the 10-day period varied by less than 0.04 between the sets. We interpret the second peak as a product of the sampling of our observations.

We performed this same analysis but instead kept the RV amplitude constant at $30 \mathrm{~km} \mathrm{~s}^{-1}$, where $\mathrm{RV}$ amplitude is defined as $\left(R V_{\max }-R V_{\min }\right) / 2$. To do this, we changed the 

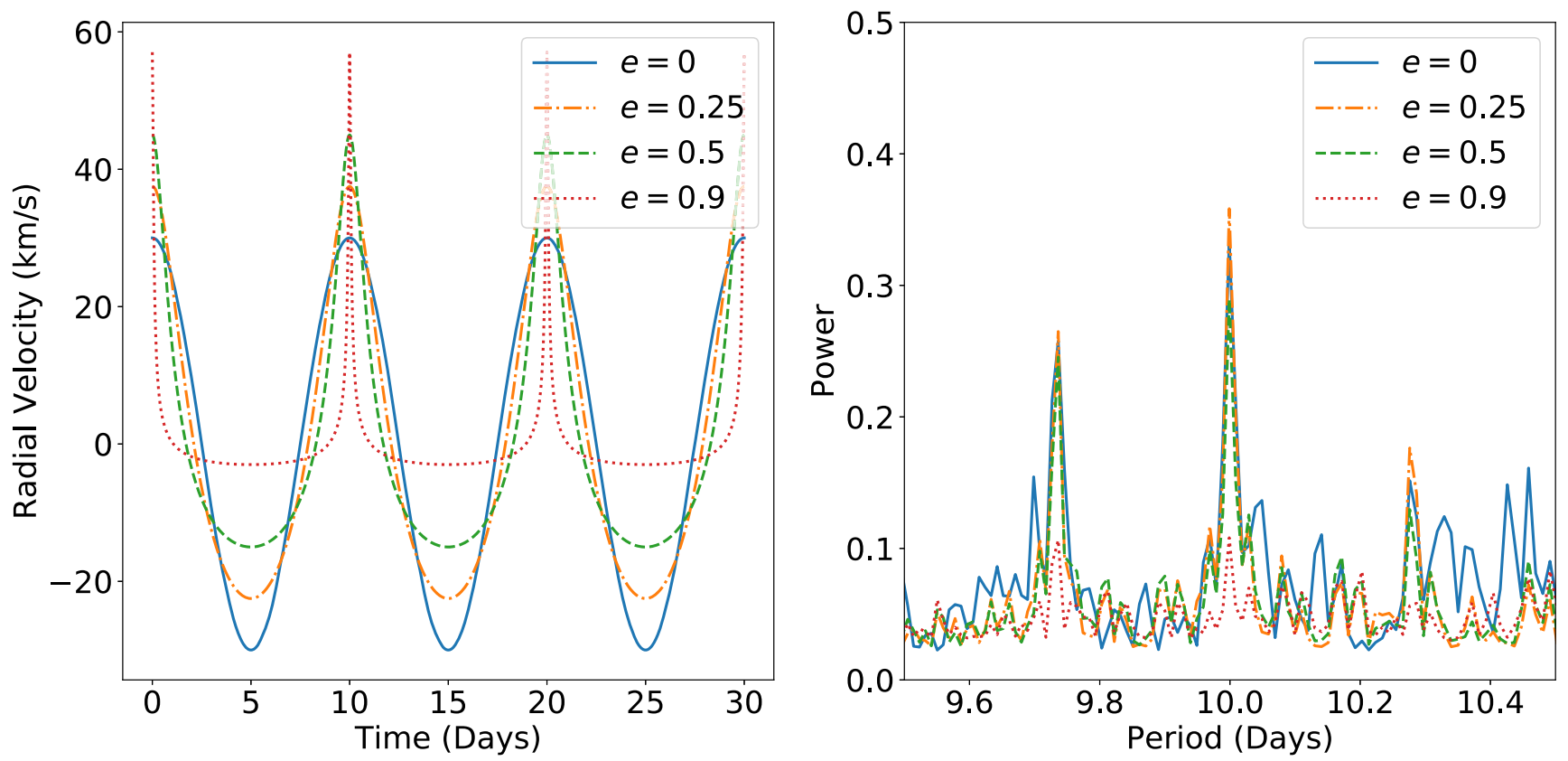

Figure 7. Left: sample radial velocity curves with different eccentricities. Each curve has a period of 10 days, an RV amplitude of $30 \mathrm{~km} \mathrm{~s}^{-1}$, and a $\omega$ of $0^{\circ}$. Each curve was sampled at the same times as our data. Right: median Lomb-Scargle power values for each set of 100,000 simulations run for the different eccentric curves.

value of $K$ for each value of eccentricity, which corresponds to changing the semimajor axis, since the period remains constant at 10 days (Figure 7). We find that the Lomb-Scargle powers at 10 days for the different eccentricities differ by less than 0.07 for eccentricities of $0,0.25$, and 0.5 . For an eccentricity of 0.9 , the power value drops to 0.11 at 10 days. We again interpret the second peak as a product of the sampling of our observations.

\section{ORCID iDs}

Andrea Ghez (i) https://orcid.org/0000-0003-3230-5055

Smadar Naoz (ib https://orcid.org/0000-0002-9802-9279

Gunther Witzel (1) https://orcid.org/0000-0003-2618-797X

Jessica R. Lu (1) https://orcid.org/0000-0001-9611-0009

\section{References}

Alexander, T. 2005, PhR, 419, 65

Angélil, R., \& Saha, P. 2010, ApJ, 711, 157

Angélil, R., \& Saha, P. 2011, ApJL, 734, L19

Astropy Collaboration, Robitaille, T. P., Tollerud, E. J., Greenfield, P., et al. 2013, A\&A, 558, A33

Bartko, H., Martins, F., Trippe, S., et al. 2009, ApJ, 708, 834

Boehle, A., Ghez, A. M., Schödel, R., et al. 2016, ApJ, 830, 17

Borka, D., Jovanović, P., Borka Jovanović, V., \& Zakharov, A. F. 2013, JCAP, 11,50

Chen, X., \& Amaro-Seoane, P. 2014, ApJL, 786, L14

Do, T., Ghez, A. M., Morris, M. R., et al. 2009, ApJ, 703, 1323

Do, T., Lu, J. R., Ghez, A. M., et al. 2013, ApJ, 764, 154

Dworetsky, M. M. 1983, MNRAS, 203, 917

Eisenhauer, F., Genzel, R., Alexander, T., et al. 2005, ApJ, 628, 246

Feroz, F., \& Hobson, M. P. 2008, MNRAS, 384, 449

Feroz, F., Hobson, M. P., \& Bridges, M. 2009, MNRAS, 398, 1601

Feroz, F., Hobson, M. P., Cameron, E., \& Pettitt, A. N. 2013, arXiv:1306.2144

Ghez, A. M., Duchêne, G., Matthews, K., et al. 2003, ApJL, 586, L127

Ghez, A. M., Salim, S., Hornstein, S. D., et al. 2005, ApJ, 620, 744

Ghez, A. M., Salim, S., Weinberg, N. N., et al. 2008, ApJ, 689, 1044

Gillessen, S., Eisenhauer, F., Fritz, T. K., et al. 2009a, ApJL, 707, L114

Gillessen, S., Eisenhauer, F., Trippe, S., et al. 2009b, ApJ, 692, 1075

Gillessen, S., Plewa, P. M., Eisenhauer, F., et al. 2017, ApJ, 837, 30

Habibi, M., Gillessen, S., Martins, F., et al. 2017, ApJ, 847, 120

Hees, A., Do, T., Ghez, A. M., et al. 2017, PhRvL, 118, 211101
Hees, A., Ghez, A. M., Do, T., et al. 2017, arXiv:1705.10792

Hilditch, R. W. 2001, An Introduction to Close Binary Stars, Vol. 392, (Cambridge: Cambridge Univ. Press)

Hills, J. G. 1988, Natur, 331, 687

Hopman, C. 2009, ApJ, 700, 1933

Iorio, L. 2017, MNRAS, 472, 2249

Johannsen, T. 2016a, CQGra, 33, 113001

Johannsen, T. 2016b, CQGra, 33, 124001

Kerr, F. J., \& Lynden-Bell, D. 1986, MNRAS, 221, 1023

Larkin, J., Barczys, M., Krabbe, A., et al. 2006, NewAR, 50, 362

Levin, Y. 2007, MNRAS, 374, 515

Li, G., Ginsburg, I., Naoz, S., \& Loeb, A. 2017, ApJ, 851, 131

Löckmann, U., Baumgardt, H., \& Kroupa, P. 2008, ApJL, 683, L151

Lomb, N. R. 1976, Ap\&SS, 39, 447

Lu, J. R., Ghez, A. M., Hornstein, S. D., et al. 2009, ApJ, 690, 1463

Madigan, A.-M., Pfuhl, O., Levin, Y., et al. 2014, ApJ, 784, 23

Martins, F., Gillessen, S., Eisenhauer, F., et al. 2008, ApJL, 672, L119

Martins, F., Trippe, S., Paumard, T., et al. 2006, ApJL, 649, L103

Merritt, D., Gualandris, A., \& Mikkola, S. 2009, ApJL, 693, L35

Morris, M. 1993, ApJ, 408, 496

Naoz, S. 2016, ARA\&A, 54, 441

Ott, T., Eckart, A., \& Genzel, R. 1999, ApJ, 523, 248

Paumard, T., Genzel, R., Martins, F., et al. 2006, ApJ, 643, 1011

Perets, H. B., Gualandris, A., Kupi, G., Merritt, D., \& Alexander, T. 2009, ApJ, 702,884

Perets, H. B., Hopman, C., \& Alexander, T. 2007, ApJ, 656, 709

Pfuhl, O., Alexander, T., Gillessen, S., et al. 2014, ApJ, 782, 101

Phifer, K., Do, T., Meyer, L., et al. 2013, ApJL, 773, L13

Psaltis, D., Wex, N., \& Kramer, M. 2016, ApJ, 818, 121

Rafelski, M., Ghez, A. M., Hornstein, S. D., Lu, J. R., \& Morris, M. 2007, ApJ, 659,1241

Rubilar, G. F., \& Eckart, A. 2001, A\&A, 374, 95

Sana, H., de Mink, S. E., de Koter, A., et al. 2012, Sci, 337, 444

Scargle, J. D. 1982, ApJ, 263, 835

Stellingwerf, R. F. 1972, A\&A, 21, 91

Stephan, A. P., Naoz, S., Ghez, A. M., et al. 2016, MNRAS, 460, 3494

Will, C. M. 2008, ApJL, 674, L25

Witzel, G., Ghez, A. M., Morris, M. R., et al. 2014, ApJL, 796, L8

Witzel, G., Sitarski, B. N., Ghez, A. M., et al. 2017, ApJ, 847, 80

Yu, Q., \& Tremaine, S. 2003, ApJ, 599, 1129

Zakharov, A. F., Jovanović, P., Borka, D., \& Borka Jovanović, V. 2016, JCAP, 05, 045

Zucker, S., Alexander, T., Gillessen, S., Eisenhauer, F., \& Genzel, R. 2006, ApJL, 639, L21 